\title{
Vedolizumab versus Adalimumab for Moderate-to-Severe Ulcerative Colitis
}

\author{
Bruce E. Sands, M.D., Laurent Peyrin-Biroulet, M.D., Ph.D., Edward V. Loftus, Jr., M.D., \\ Silvio Danese, M.D., Jean-Frédéric Colombel, M.D., Murat Törüner, M.D., \\ Laimas Jonaitis, M.D., Ph.D., Brihad Abhyankar, F.R.C.S., Jingjing Chen, Ph.D., \\ Raquel Rogers, M.D., Richard A. Lirio, M.D., Jeffrey D. Bornstein, M.D., and \\ Stefan Schreiber, M.D., Ph.D., for the VARSITY Study Group**
}

\section{BACKGROUND}

Biologic therapies are widely used in patients with ulcerative colitis. Head-to-head trials of these therapies in patients with inflammatory bowel disease are lacking.

\section{METHODS}

In a phase $3 \mathrm{~b}$, double-blind, double-dummy, randomized, active-controlled trial conducted at 245 centers in 34 countries, we compared vedolizumab with adalimumab in adults with moderately to severely active ulcerative colitis to determine whether vedolizumab was superior. Previous exposure to a tumor necrosis factor inhibitor other than adalimumab was allowed in up to $25 \%$ of patients. The patients were assigned to receive intravenous infusions of $300 \mathrm{mg}$ of vedolizumab on day 1 and at weeks 2, 6, 14, 22, 30, 38, and 46 (plus injections of placebo) or subcutaneous injections of $40 \mathrm{mg}$ of adalimumab, with a total dose of $160 \mathrm{mg}$ at week $1,80 \mathrm{mg}$ at week 2, and $40 \mathrm{mg}$ every 2 weeks thereafter until week 50 (plus infusions of placebo). Dose escalation was not permitted in either group. The primary outcome was clinical remission at week 52 (defined as a total score of $\leq 2$ on the Mayo scale [range, 0 to 12, with higher scores indicating more severe disease] and no subscore $>1$ [range, 0 to 3] on any of the four Mayo scale components). To control for type I error, efficacy outcomes were analyzed with the use of a hierarchical testing procedure, with the variables in the following order: clinical remission, endoscopic improvement (subscore of 0 to 1 on the Mayo endoscopic component), and corticosteroid-free remission at week 52.

RESULTS

A total of 769 patients underwent randomization and received at least one dose of vedolizumab (383 patients) or adalimumab (386 patients). At week 52, clinical remission was observed in a higher percentage of patients in the vedolizumab group than in the adalimumab group (31.3\% vs. $22.5 \%$; difference, 8.8 percentage points; $95 \%$ confidence interval $[\mathrm{CI}], 2.5$ to $15.0 ; \mathrm{P}=0.006)$, as was endoscopic improvement $(39.7 \%$ vs. $27.7 \%$; difference, 11.9 percentage points; $95 \%$ CI, 5.3 to 18.5; P<0.001). Corticosteroid-free clinical remission occurred in $12.6 \%$ of the patients in the vedolizumab group and in $21.8 \%$ in the adalimumab group (difference, -9.3 percentage points; $95 \% \mathrm{CI},-18.9$ to 0.4). Exposure-adjusted incidence rates of infection were 23.4 and 34.6 events per 100 patient-years in the vedolizumab group and adalimumab group, respectively, and the corresponding rates for serious infection were 1.6 and 2.2 events per 100 patient-years.

\section{CONCLUSIONS}

In this trial involving patients with moderately to severely active ulcerative colitis, vedolizumab was superior to adalimumab with respect to achievement of clinical remission and endoscopic improvement, but not corticosteroid-free clinical remission. (Funded by Takeda; VARSITY ClinicalTrials.gov number, NCT02497469; EudraCT number, 2015-000939-33.)
From the Icahn School of Medicine at Mount Sinai, New York (B.E.S., J.-F.C.); Nancy University Hospital, Nancy, France (L.P.-B.); Mayo Clinic College of Medicine, Rochester, MN (E.V.L.); Humanitas University, Milan (S.D.); Ankara University School of Medicine, Ankara, Turkey (M.T.); Lithuanian University of Health Sciences, Kaunas, Lithuania (L.J.); Takeda Development Centre Europe, London (B.A.); Takeda Development Center Americas, Cambridge, MA (J.C., R.R., R.A.L., J.D.B.); and the University Hospital Schleswig-Holstein, Kiel, Germany (S.S.). Address reprint requests to Dr. Sands at the Division of Gastroenterology, Icahn School of Medicine at Mount Sinai, 1 Gustave L. Levy PI., Box 1069, New York, NY 10029, or at bruce .sands@mssm.edu; or to Dr. Schreiber at the Department of Medicine I, University Hospital Schleswig-Holstein, RosalindFranklin-Str. 12, Haus 5, 24105 Kiel, Germany, or at s.schreiber@mucosa.de.

*A list of investigators in the VARSITY Study Group is provided in the Supplementary Appendix, available at NEJM.org.

Drs. Sands and Schreiber contributed equally to this article.

This article was updated on September 26, 2019, at NEJM.org.

N Engl J Med 2019;381:1215-26. DOI: 10.1056/NEJMoa1905725 Copyright (C) 2019 Massachusetts Medical Society. 
U LCERATIVE COLITIS IS A CHRONIC INflammatory disorder of the large bowel characterized by abdominal pain, bloody diarrhea, and fecal urgency. ${ }^{1}$ Agents that are commonly used when conventional treatments (e.g., aminosalicylates, oral immunomodulators, and corticosteroids) fail include tofacitinib, a smallmolecule Janus kinase inhibitor, and biologic agents, such as tumor necrosis factor (TNF) inhibitors (e.g., infliximab, adalimumab, and golimumab) and vedolizumab, an anti-integrin antibody. ${ }^{2,3}$ These medications were shown to be effective in randomized, placebo-controlled trials, but whereas head-to-head trials that directly compare agents have been performed in patients with rheumatologic diseases, few such trials have been performed in patients with inflammatory bowel disease. ${ }^{4,5}$

Adalimumab, a humanized monoclonal antibody that binds and neutralizes TNF, is widely used to treat ulcerative colitis. The gut-selective, anti-integrin vedolizumab is a humanized monoclonal antibody that specifically binds to the leukocyte integrin $\alpha_{4} \beta_{7^{\circ}}{ }^{6,7}$ Here we report the results of the VARSITY trial, which directly compared the efficacy and safety of vedolizumab with those of adalimumab in patients with moderately to severely active ulcerative colitis.

METHODS

\section{TRIAL DESIGN}

The VARSITY trial, a phase $3 \mathrm{~b}$, randomized, double-blind, double-dummy, active-controlled superiority trial to detect treatment differences between vedolizumab and adalimumab, was conducted from July 2015 through January 2019 at 245 sites in 34 countries. (For details on the trial design, eligibility criteria, assessments, outcome measures, and statistical analyses, see the Supplementary Appendix, available with the full text of this article at NEJM.org.) The trial protocol, available at NEJM.org, was approved by an institutional review board or ethics committee at each trial site, and all the patients provided written informed consent.

\section{PATIENTS}

Adults 18 to 85 years of age were eligible for inclusion in the trial if they had moderately to severely active ulcerative colitis, defined as a total score of 6 to 12 on the Mayo scale ${ }^{8}$ (total Mayo scores range from 0 to 12 , with higher scores indicating more severe disease) and a subscore of at least 2 on the endoscopic component of the Mayo scale (subscores on each of the four components of the Mayo scale range from 0 to 3); colonic involvement of at least $15 \mathrm{~cm}$; and had a confirmed diagnosis of ulcerative colitis at least 3 months before screening. Patients who had not previously used a TNF inhibitor and had no response or loss of response to conventional treatments were eligible. Patients who had discontinued treatment with a TNF inhibitor (except adalimumab) because of documented reasons other than safety were also eligible, with enrollment capped at $25 \%$. All patients had not previously received vedolizumab.

\section{SCREENING ASSESSMENTS}

Screening assessments included a physical examination, endoscopy (findings were read at a central location), the total Mayo score, blood and stool tests, tuberculosis screening, the score on the Inflammatory Bowel Disease Questionnaire (IBDQ; scores range from 32 to 224, with higher scores indicating a better quality of life), ${ }^{9}$ and a questionnaire to identify possible symptoms of progressive multifocal leukoencephalopathy.

\section{RANDOMIZATION AND TREATMENTS}

Patients who were assigned to the vedolizumab group received intravenous infusions of $300 \mathrm{mg}$ of vedolizumab on day 1 and at weeks 2, 6, 14, $22,30,38$, and 46 plus subcutaneous injections of placebo on day 1 (four injections), at week 2 (two injections), and every 2 weeks thereafter (single injections) until week 50. Patients who were assigned to the adalimumab group received multiple subcutaneous injections of $40 \mathrm{mg}$ of adalimumab on days 1 and 2 (either four injections on day 1 or two injections per day for 2 days [total dose of $160 \mathrm{mg}$ ]), two injections of $40 \mathrm{mg}$ at week 2 (total dose of $80 \mathrm{mg}$ ), and single injections of $40 \mathrm{mg}$ every 2 weeks thereafter until week 50 plus intravenous infusions of placebo at day 1 and weeks 2, 6, 14, 22, 30, 38, and 46 . Dose escalation was not permitted in either treatment group.

Randomization was performed at a central location with the use of computer-generated randomization schedules and was stratified ac- 
cording to previous use of a TNF inhibitor (yes or no) and concomitant use of an oral corticosteroid (yes or no). Among the patients who were receiving an oral corticosteroid at baseline, the dose must have been stable ( $\leq 30 \mathrm{mg}$ per day of prednisone or equivalent) for at least 2 weeks before the first dose of a trial drug. The corticosteroid dose remained unaltered through week 6 of the trial, and after week 6 , the dose was tapered intermittently if the patient had a response. Patients who had a loss of response during the tapering period were permitted to receive the baseline corticosteroid dose one time only before tapering was restarted. In accordance with the protocol, patients in whom the corticosteroid dose could not be tapered were withdrawn from the trial and were considered to have treatment failures with respect to each of the outcomes. Patients who were not receiving corticosteroids at baseline but who initiated corticosteroid treatment during the trial were withdrawn because of lack of efficacy. Patients who were receiving an aminosalicylate or an immunomodulator at baseline maintained stable doses throughout the trial.

\section{FOLLOW-UP ASSESSMENTS}

Regular trial visits occurred through week 52, with a final safety follow-up visit at week 68 . Adverse events (classified according to the Medical Dictionary for Regulatory Activities, version 21.0), results of laboratory tests and safety assessments, and concomitant medications were recorded throughout the trial. A partial score on the Mayo scale, ${ }^{10}$ which consisted of the combined subscores (range, 0 to 9) on three of the four components of the Mayo scale (stool frequency, rectal bleeding, and physician's global assessment, with the exclusion of endoscopy), was calculated at weeks 2, 4, 6, 22, 30, 38, and 46. The total Mayo score was calculated at weeks 14 and 52, when endoscopy was performed. Measurements of the fecal calprotectin level were performed at weeks 14, 30, and 52. IBDQ assessments were performed at weeks 30 and 52 .

\section{OUTCOME MEASURES}

The primary outcome was clinical remission at week 52 (defined as a total score of $\leq 2$ on the Mayo scale and no subscore $>1$ on any of the four components). Secondary outcomes were endoscopic improvement (defined as a subscore of 0 or 1 on the Mayo endoscopic component [originally termed "mucosal healing" in the protocol]) and corticosteroid-free clinical remission at week 52, which was assessed only in patients who were receiving corticosteroids at baseline.

There were 42 prespecified outcomes (26 were prespecified in the trial protocol and another 16 in the statistical analysis plan [available with the protocol]). All outcomes other than the primary and two secondary outcomes were referred to as "additional end points" in the protocol and statistical analysis plan and are considered to be exploratory outcomes. These prespecified exploratory outcomes included durable clinical remission (defined as clinical remission at both week 14 and week 52); improvement in the subscores on the patient-reported components of the Mayo scale (stool frequency and rectal bleeding); improvement in quality of life (defined as an increase of $\geq 16$ points in IBDQ score); histologic remission (defined as a Geboes score $<2.0$ [on a scale from 0 to 5.4, with higher scores indicating more severe disease activity] and a Robarts Histopathology Index score $<3$ [on a scale from 0 to 33 , with higher scores indicating more severe disease activity] $)^{11,12}$; minimal histologic disease (defined as a Geboes score $<3.2$ and a Robarts Histopathology Index score $<5$ ); clinical response (defined as a reduction in the partial Mayo score [stool frequency, rectal bleeding, and physician's global assessment] of $\geq 2$ points and of $\geq 25 \%$ from baseline, with an accompanying decrease in rectal bleeding subscore of $\geq 1$ point or absolute rectal bleeding subscore of $\leq 1$ point); and safety (as assessed by the incidence of adverse events).

\section{TRIAL OVERSIGHT}

The trial sponsor (Takeda) designed the trial in conjunction with the principal academic investigators and provided the trial drugs and placebo. A clinical research organization (IQVIA), funded by the sponsor, managed all the collection of the data, maintained the trial database in a blinded manner, and performed the data analyses. The trial investigators, the participating institutions, the clinical research organization, and the sponsor agreed to maintain data confidentiality. The initial draft of the manuscript was written by one of the authors employed by the sponsor in collaboration with the first and last authors. A medical 
writer, funded by the sponsor, assisted with the preparation of subsequent drafts. All the authors interpreted the data, contributed to subsequent drafts, and made the decision to submit the manuscript for publication. The academic authors had access to the data and vouch for the accuracy and completeness of the data and for the fidelity of the trial to the protocol.

\section{STATISTICAL ANALYSIS}

Efficacy was analyzed according to treatment randomization group in the full-analysis set, which included all patients who underwent randomization and received at least one dose of a trial drug. Adverse events were analyzed according to the treatment actually received in the safety population, which included all the patients in the full-analysis set. Missing values for binary outcomes were imputed as nonresponses, and missing values for continuous outcomes were imputed with the use of the last-observationcarried-forward approach. We performed a prespecified sensitivity analysis of the primary efficacy outcome that used a hybrid imputation approach to assess the effect of discontinuation under different missing data mechanisms. First, under the assumption that data were not missing at random, missing data for patients who discontinued vedolizumab or adalimumab because of an adverse event or lack of efficacy were imputed as nonresponses. Second, under the assumption that data were missing at random, data that were missing for other reasons were imputed with the use of multiple imputation. This sensitivity analysis was repeated post hoc for the two secondary efficacy outcomes.

In the primary efficacy analysis, we compared the treatment groups with respect to the percentages of patients who had clinical remission at week 52 using the conventional CochranMantel-Haenszel chi-square test, with adjustment for the randomization stratification factors. A hierarchical closed-testing procedure was used to control the inflation of the type I error rate due to multiple efficacy outcomes. Efficacy outcomes were tested in the following order: clinical remission at week 52, endoscopic improvement at week 52, and corticosteroid-free clinical remission at week 52 (two-tailed $\mathrm{P}<0.05$ was required to proceed to the next test).

In a post hoc analysis, we assessed efficacy using the weighted statistical method described by Cui et al. ${ }^{13}$ to provide strong control of the familywise type I error rate in the presence of interim sample-size reestimation. Efficacy was also assessed in prespecified subgroup analyses that were performed on the basis of disease characteristics and previous use of a TNF inhibitor (yes or no). The exposure-adjusted incidence rate (per 100 patient-years) was defined as the number of patients who had the adverse event divided by the total exposure time among the patients. The extent of exposure for each patient was calculated as the duration between the first and last dose of a trial drug plus approximately five times the half-life of the drug.

We estimated that a sample size of 329 patients per treatment group would provide the trial with $86 \%$ power to detect a significant difference in clinical remission at week 52 (two-tailed chi-square test at $\mathrm{P}<0.05$ ), assuming that remission would occur in $28 \%$ of the patients in the vedolizumab group and in 18\% in the adalimumab group. We also estimated that this sample size would provide the trial with $80 \%$ power to detect differences in endoscopic improvement at week 52 (two-tailed chi-square test at $\mathrm{P}<0.05$ ), assuming that improvement would occur in $35 \%$ of the patients in the vedolizumab group and in $25 \%$ in the adalimumab group. The sample size was increased by 100 patients after we performed a prespecified adaptive sample-size reestimation using the promising zone design. ${ }^{14}$

\section{RESULTS}

\section{PATIENT CHARACTERISTICS}

A total of 1285 patients were screened for eligibility, and 771 were enrolled in the trial, of whom 769 underwent randomization and received at least one dose of vedolizumab (383 patients) or adalimumab (386 patients). The characteristics of the patients were generally similar between the treatment groups (Table 1). Discontinuation of treatment because of lack of efficacy occurred in 41 patients in the vedolizumab group and in 82 patients in the adalimumab group. (Also see Fig. S1 and Table S2 in the Supplementary Appendix.)

\section{EFFICACY}

\section{Clinical Remission}

Clinical remission at week 52 (primary outcome) was observed in a higher percentage of patients 
in the vedolizumab group than in the adalimumab group (31.3\% [120 of 383] vs. $22.5 \%$ [ 87 of 386], $\mathrm{P}=0.006)$ - a difference of 8.8 percentage points $(95 \%$ confidence interval [CI], 2.5 to 15.0$)$ after adjustment with the Cochran-MantelHaenszel test (Fig. 1A). A sensitivity analysis to evaluate the effect of withdrawals showed that clinical remission at week 52 occurred in 37.2\% of the patients in the vedolizumab group and in $25.9 \%$ in the adalimumab group (adjusted difference, 11.3 percentage points; $95 \%$ CI, 4.6 to 18.0). (See Tables S3 and S4 in the Supplementary Appendix.)

Among the patients who had not previously used a TNF inhibitor, clinical remission at week 52 was observed in $34.2 \%$ in the vedolizumab group and in $24.3 \%$ in the adalimumab group; among the patients who had previous exposure to a TNF inhibitor other than adalimumab, the corresponding percentages were $20.3 \%$ and $16.0 \%$ (Fig. 1A). The treatment effects in other subgroups defined according to demographic and disease characteristics were generally consistent with those in the overall population (Fig. S2 in the Supplementary Appendix; Fig. S3 provides the percentages of patients who had clinical remission at week 52 among those who were receiving an oral corticosteroid or immunomodulator at baseline and among those who were not).

At week 14, clinical remission in the overall population was observed in $26.6 \%$ of the patients (102 of 383) in the vedolizumab group and in $21.2 \%$ ( 82 of 386) in the adalimumab group (difference, 5.3 percentage points; 95\% CI, -0.7 to 11.4) (Fig. S4 in the Supplementary Appendix). Durable clinical remission occurred in $18.3 \%$ of the patients (70 of 383) in the vedolizumab group and in $11.9 \%$ (46 of 386) in the adalimumab group (difference, 6.3 percentage points; 95\% CI, 1.3 to 11.3 ).

\section{Endoscopic Improvement}

At week 52, endoscopic improvement (first secondary outcome) was observed in a higher percentage of patients in the vedolizumab group than in the adalimumab group (39.7\% [152 of 383] vs. $27.7 \%$ [107 of 386], $\mathrm{P}<0.001)-$ a difference of 11.9 percentage points $(95 \%$ CI, 5.3 to 18.5) after adjustment with the Cochran-MantelHaenszel test (Fig. 1B). A sensitivity analysis to evaluate the effect of withdrawals showed that

\begin{tabular}{|c|c|c|}
\hline Characteristic & $\begin{array}{l}\text { Adalimumab } \\
(\mathrm{N}=386)\end{array}$ & $\begin{array}{l}\text { Vedolizumab } \\
\quad(\mathrm{N}=385)\end{array}$ \\
\hline Age $-y r$ & $40.5 \pm 13.4$ & $40.8 \pm 13.7$ \\
\hline Male sex — no. (\%) & $216(56.0)$ & $234(60.8)$ \\
\hline White race — no. (\%) $†$ & $341(88.3)$ & $345(89.6)$ \\
\hline Body weight — kg & $73.4 \pm 18.4$ & $72.7 \pm 17.0$ \\
\hline Current smoker - no. (\%) $\ddagger$ & $23(6.0)$ & $19(4.9)$ \\
\hline Duration of ulcerative colitis - yr $\int$ & $6.4 \pm 6.0$ & $7.3 \pm 7.2$ \\
\hline Total score on the Mayo scaleq & $8.7 \pm 1.5$ & $8.7 \pm 1.6$ \\
\hline Fecal calprotectin level $-\mu \mathrm{g} / \mathrm{g} \|$ & $2771 \pm 4064$ & $2929 \pm 5920$ \\
\hline $\begin{array}{l}\text { Previous treatment with a TNF inhibitor } \\
\text { with documented reason for } \\
\text { discontinuation - no. (\%) }\end{array}$ & $81(21.0)$ & $80(20.8)$ \\
\hline $\begin{array}{c}\text { Previous therapy with a TNF inhibitor with } \\
\text { documented failure - no. (\%) }\end{array}$ & $79(20.5)$ & $72(18.7)$ \\
\hline Inadequate response & $40(50.6)$ & $36(50.0)$ \\
\hline Loss of response & $29(36.7)$ & $24(33.3)$ \\
\hline Side effects & $3(3.8)$ & $7(9.7)$ \\
\hline Missing data & $7(8.9)$ & $5(6.9)$ \\
\hline \multicolumn{3}{|l|}{$\begin{array}{c}\text { Concomitant use of medications for } \\
\text { ulcerative colitis - no. (\%) }\end{array}$} \\
\hline Corticosteroids only*** & $140(36.3)$ & $139(36.1)$ \\
\hline Immunomodulators only†' & $100(25.9)$ & $101(26.2)$ \\
\hline
\end{tabular}

* Plus-minus values are means \pm SD. TNF denotes tumor necrosis factor.

$\dagger$ Race was reported by the patient.

Data on smoking status were missing for two patients in the vedolizumab group.

$\int$ One patient in the adalimumab group had ulcerative colitis of unknown duration.

9 The total score on the Mayo scale ranges from 0 to 12, with a higher score indicating more active disease. The four components of the Mayo scale are stool frequency, rectal bleeding, endoscopy (sigmoidoscopy), and physician's global assessment. Total Mayo scores were available for 384 patients in the adalimumab group and 380 patients in the vedolizumab group.

\| Data on the fecal calprotectin level were available for 332 patients in the adalimumab group and 341 patients in the vedolizumab group.

$* *$ Concomitant use of corticosteroids was determined according to the report in the interactive Web response system.

ti Concomitant use of immunomodulators was determined according to the report in the electronic case-report form. The commonly used immunomodulators, in order from most to least used, were azathioprine, mercaptopurine, and methotrexate.

endoscopic improvement at week 52 occurred in $46.8 \%$ of the patients in the vedolizumab group and in $33.6 \%$ in the adalimumab group (adjusted difference, 13.2 percentage points; $95 \% \mathrm{CI}, 6.0$ to 20.3). Among the patients who had not previously used a TNF inhibitor, endoscopic improvement at week 52 occurred in $43.1 \%$ in the vedolizumab group and in $29.5 \%$ in the adalimumab group, and among the patients who had previ- 


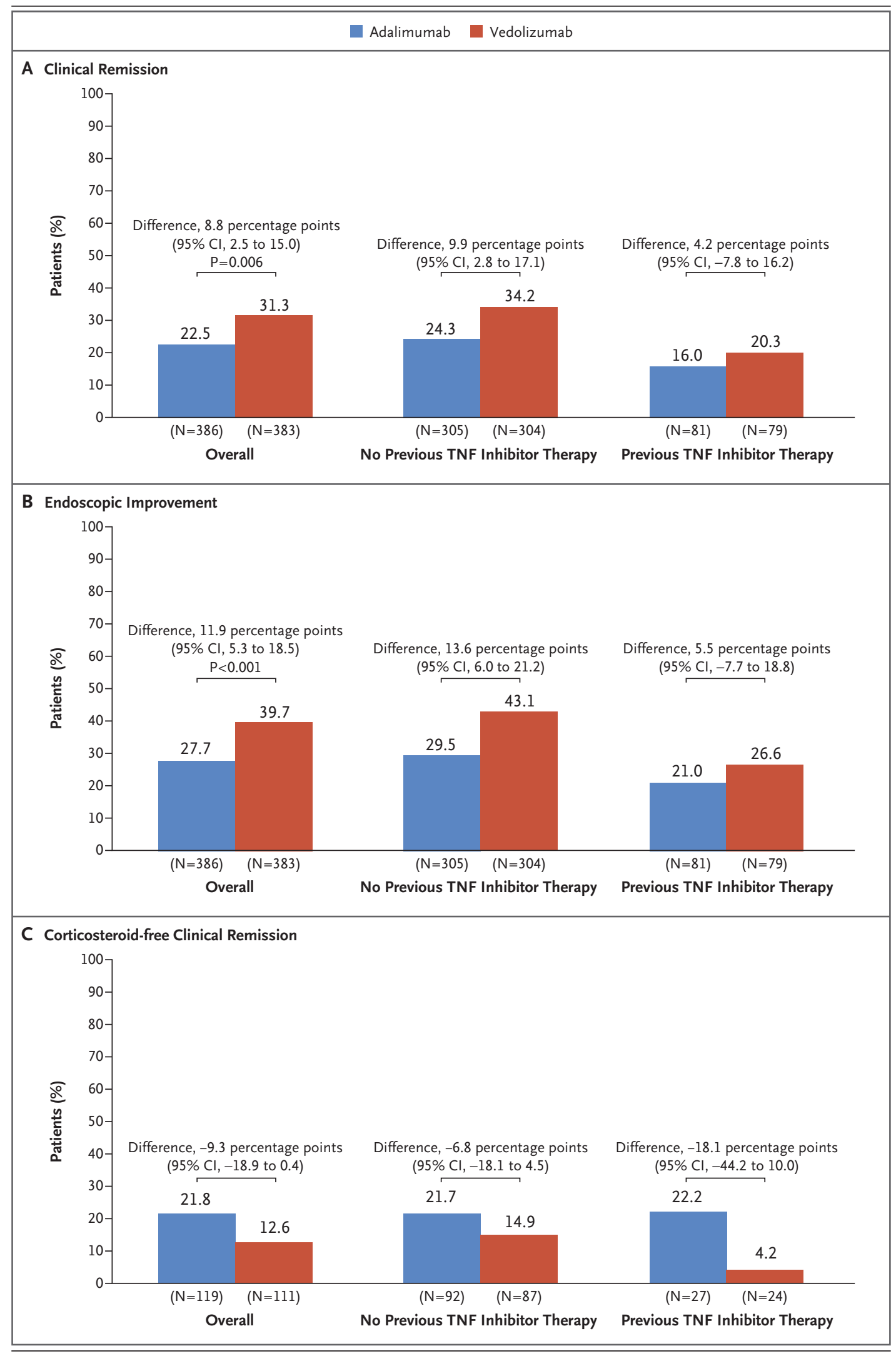


Figure 1 (facing page). Efficacy Outcomes at Week 52 in the Full-Analysis Set and in Subgroups Defined According to Previous Treatment with a TNF Inhibitor.

Shown are the percentages of patients who had clinical remission at week 52 (Panel A), endoscopic improvement at week 52 (Panel B), and corticosteroid-free remission at week 52 (Panel C). Efficacy was analyzed according to treatment randomization group in the full-analysis set, which included all patients who underwent random ization and received at least one dose of a trial drug. The subgroup of patients with no previous tumor necrosis factor (TNF) inhibitor therapy included those who had not previously used a TNF inhibitor and had no response or loss of response to conventional treatments; the subgroup of patients with previous TNF inhibitor therapy included those who had previous exposure to a TNF inhibitor other than adalimumab and had a documented reason for discontinuation of the therapy other than safety. The analysis of corticosteroid-free clinical remission was performed only in the subgroup of patients who were receiving corticosteroids at baseline (as determined from the electronic case report form). Point estimates, $95 \%$ confidence intervals, and $P$ values were calculated with the use of the CochranMantel-Haenszel chi-square test, with adjustment for the randomization stratification factors, or with the use of Fisher's exact method if the numerator was five patients or fewer.

ous exposure to a TNF inhibitor other than adalimumab, the corresponding percentages were $26.6 \%$ and $21.0 \%$ (Fig. 1B). The treatment effects in the other subgroups defined according to demographic and disease characteristics were generally consistent with those in the overall population (Fig. S5 in the Supplementary Appendix). (See Fig. S3 and Tables S3 through S5 in the Supplementary Appendix.)

\section{Corticosteroid-free Clinical Remission}

At week 52, corticosteroid-free clinical remission (second secondary outcome) was observed in $12.6 \%$ of the patients (14 of 111 ) in the vedolizumab group and in $21.8 \%$ (26 of 119) in the adalimumab group (difference, -9.3 percentage points; $95 \%$ CI, -18.9 to 0.4 ) (Fig. 1C). A sensitivity analysis to evaluate the effect of withdrawals showed that corticosteroid-free clinical remission at week 52 occurred in $16.9 \%$ of the patients in the vedolizumab group and in $24.7 \%$ in the adalimumab group (adjusted difference, -7.8 percentage points; $95 \% \mathrm{CI},-18.8$ to 3.1 ). The treatment effects in the other subgroups defined according to demographic and disease characteristics were generally consistent with those in the overall population. (See Table S4 and Fig. S6 in the Supplementary Appendix.)

The median change in the oral corticosteroid dose from baseline to week 52 was $-10.0 \mathrm{mg}$ in the vedolizumab group and $-7.0 \mathrm{mg}$ in the adalimumab group. The median corticosteroid dose at week 52 was $0 \mathrm{mg}$ (range, 0 to 40 ) in the vedolizumab group and $2.5 \mathrm{mg}$ (range, 0 to 70 ) in the adalimumab group. (For absolute mean reductions in oral corticosteroid doses, see Fig. S7 in the Supplementary Appendix.)

\section{Patient-Reported Outcomes}

The percentage of patients who were in clinical remission at week 52 and who also had a subscore of 0 on both the rectal bleeding and endoscopic components of the Mayo scale was $22.2 \%$ (85 of 383) in the vedolizumab group and $14.0 \%$ (54 of 386) in the adalimumab group (difference, 8.2 percentage points; $95 \%$ CI, 2.8 to 13.5 ). In addition, $58.2 \%$ of the patients ( 223 of 383 ) in the vedolizumab group had a subscore of 0 or 1 on the stool frequency component of the Mayo scale at week 52, as compared with $44.8 \%$ (173 of 386) in the adalimumab group (difference, 13.3 percentage points; 95\% CI, 6.4 to 20.3). (For patientreported outcomes, see Table S5 in the Supplementary Appendix.)

\section{Patient Quality of Life}

Quality of life improved from baseline to week 52 (as indicated by an increase of $\geq 16$ points in the IBDQ score) in $52.0 \%$ of the patients in the vedolizumab group and in $42.2 \%$ in the adalimumab group (difference, 9.7 percentage points; 95\% CI, 2.7 to 16.7). Patient-assessed improvement at week 52 (defined as a score $>170$ on the IBDQ) was reported in $50.1 \%$ of the patients in the vedolizumab group and in $40.4 \%$ in the adalimumab group (difference, 9.6 percentage points; 95\% CI, 2.8 to 16.5). (See Table S5 in the Supplementary Appendix.)

\section{Histologic Remission}

Histologic remission at week 52 (as indicated by a Geboes score $<2.0$ ) occurred in $10.4 \%$ of the patients (40 of 383) in the vedolizumab group and in $3.1 \%$ (12 of 386 ) in the adalimumab group (difference, 7.3 percentage points; $95 \% \mathrm{CI}$, 3.8 to 10.8) (Fig. 2A). The results were similar for histologic remission as indicated by a Robarts Histopathology Index score lower than 3 (Fig. 2B). 


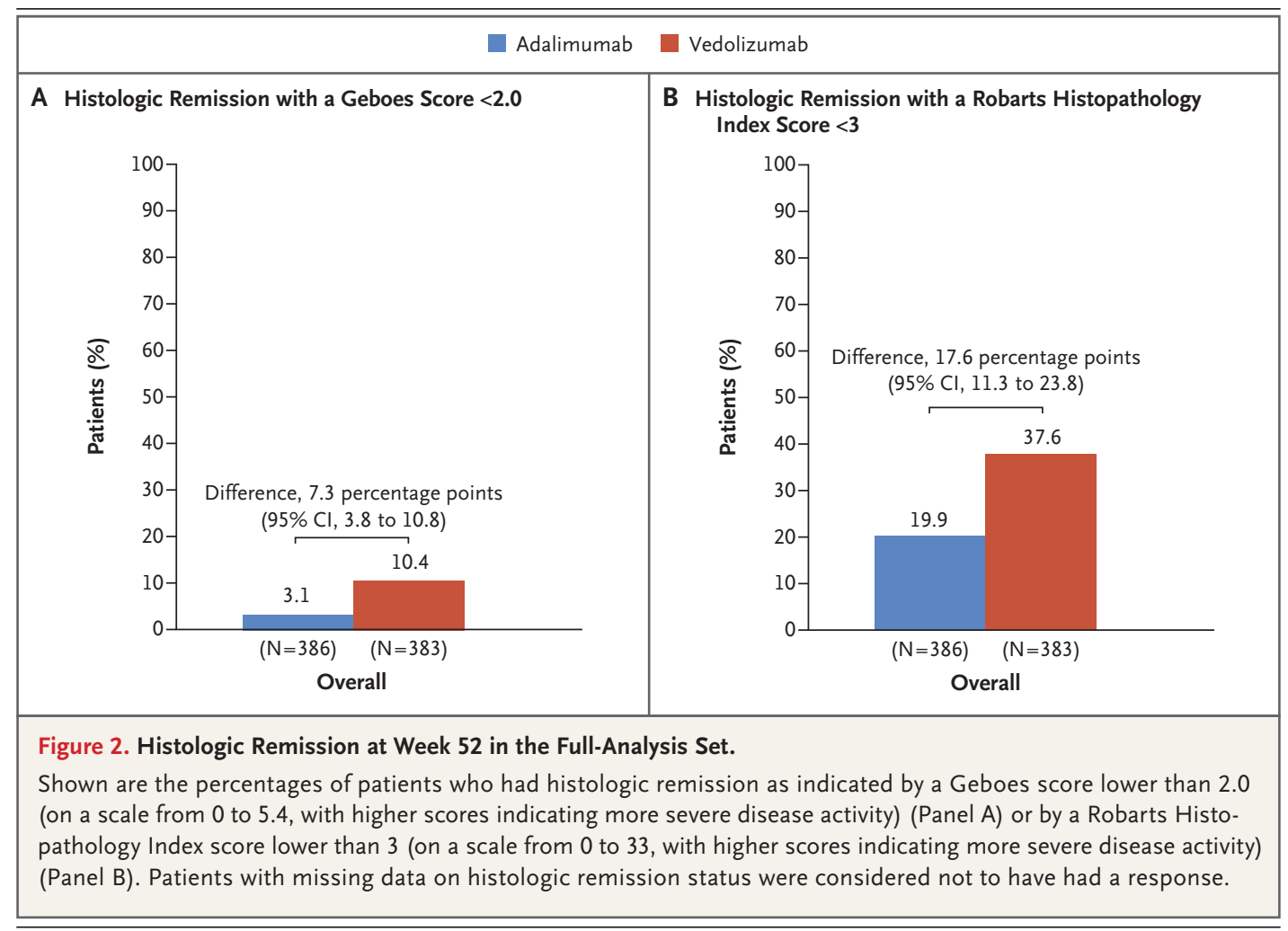

(Histologic remission at week 52 in the subgroups of patients defined according to previous treatment with a TNF inhibitor is shown in Fig. S8 in the Supplementary Appendix; histologic remission at week 14 was also assessed with the Geboes score and the Robarts Histopathological Index score, shown in Fig. S9.)

Minimal histologic disease activity as indicated by a Geboes score lower than 3.2 at week 52 was observed in $33.4 \%$ of the patients in the vedolizumab group and in $13.7 \%$ in the adalimumab group (difference, 19.6 percentage points; $95 \%$ CI, 13.8 to 25.5). Minimal histologic disease activity as indicated by a Robarts Histopathology Index score lower than 5 at week 52 was observed in $42.3 \%$ of the patients in the vedolizumab group and in $25.6 \%$ in the adalimumab group (difference, 16.6 percentage points; 95\% CI, 10.0 to 23.1). (For details on minimal histologic disease activity, see Fig. S10 in the Supplementary Appendix.)

\section{Clinical Response}

The percentages of patients who had a clinical response according to the partial Mayo score are shown in Figure 3. At week 14, a clinical response according to the total Mayo score was observed in $67.1 \%$ of the patients (257 of 383) in the vedolizumab group and in $45.9 \%$ (177 of 386) in the adalimumab group (difference, 21.2 percentage points; 95\% CI, 14.4 to 28.0) (Fig. S11 in the Supplementary Appendix; results of all other prespecified outcome assessments are provided in Table S5).

\section{SAFETY}

Adverse events occurred in $62.7 \%$ of the patients (240 of 383) in the vedolizumab group and in $69.2 \%$ (267 of 386) in the adalimumab group (Table 2). The most frequent adverse events are presented in Table S6. Serious adverse events occurred in $11.0 \%$ of the patients (42 of 383) in the vedolizumab group and in $13.7 \%$ (53 of 386) in the adalimumab group (Table S7 in the Supplementary Appendix).

Exposure-adjusted incidence rates of infections and serious infections showed that both occurred less frequently with vedolizumab than with adalimumab (infections, 23.4 vs. 34.6 events per 100 patient-years; serious infections, 1.6 vs. 


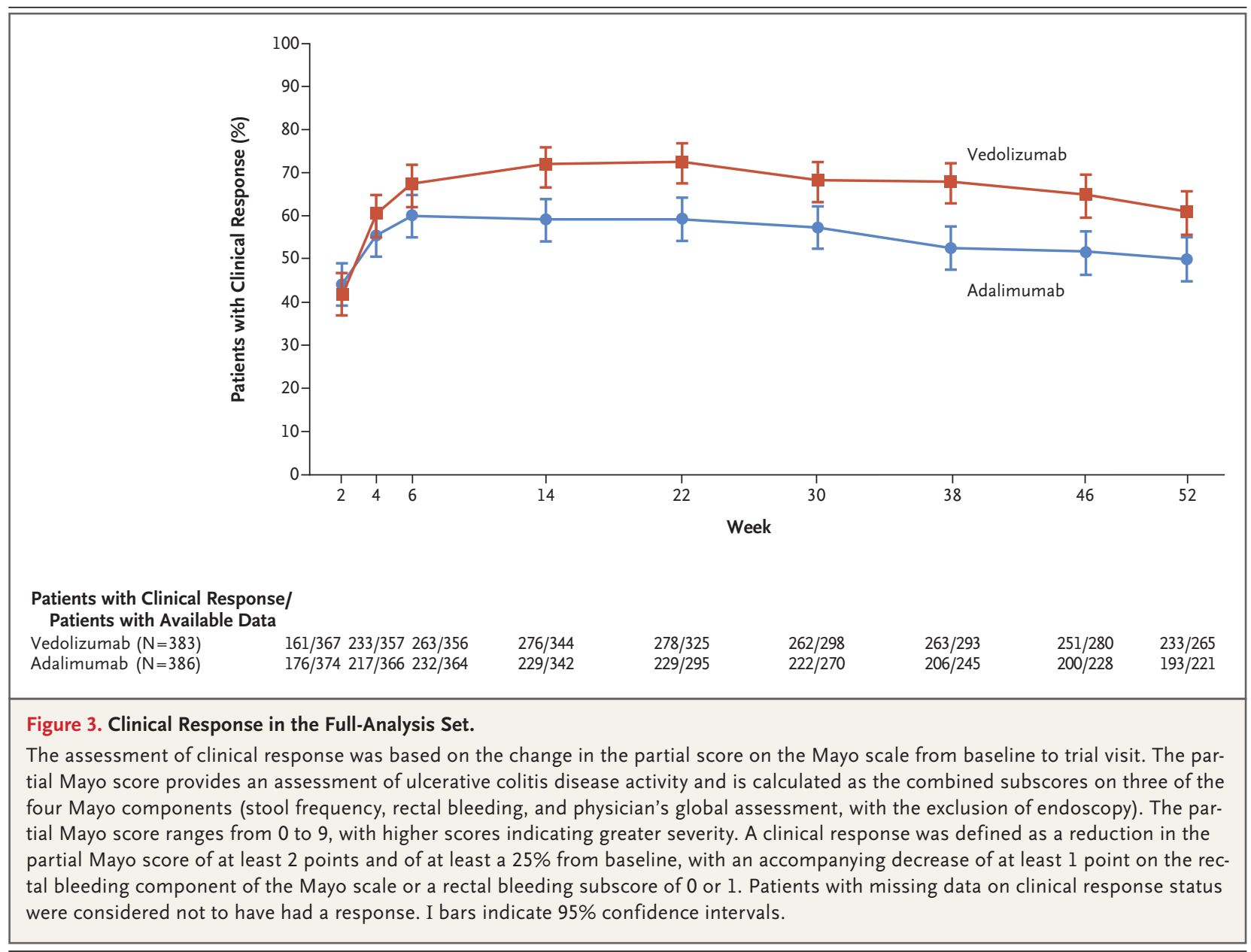

2.2 events per 100 patient-years). Herpes zoster infection was less frequent with vedolizumab than with adalimumab (0.5 vs. 4.2 per 100 patientyears), although Clostridium difficile infection was more frequent (1.1 vs. 0.6 per 100 patient-years). No patient received a diagnosis of progressive multifocal leukoencephalopathy. One patient in the vedolizumab group died because of an exacerbation of ulcerative colitis and postoperative complications that were considered by the trial site investigator to be unrelated to vedolizumab or adalimumab (Table 2).

\section{DISCUSSION}

In this comparative clinical trial of two biologic agents involving patients with moderately to severely active ulcerative colitis, clinical remission and endoscopic improvement, but not corticoste- roid-free clinical remission, were observed in a higher percentage of patients in the vedolizumab group than in the adalimumab group. In the Ulcerative Colitis Long-term Remission and Maintenance with Adalimumab 2 (ULTRA2) placebocontrolled trial, clinical remission at week 52 occurred in $17.3 \%$ of the patients in the adalimumab group and in $8.5 \%$ in the placebo group. ${ }^{15}$ As in the VARSITY trial, the ULTRA2 trial maintained blinding and randomization throughout the treatment period. In the GEMINI 1 placebocontrolled trial of vedolizumab, the percentages of patients who had clinical remission at week 52 were higher ( $41.8 \%$ of the patients in the vedolizumab group vs. $15.9 \%$ in the placebo group). ${ }^{16}$ The higher percentages of patients who had a response to active therapy in the GEMINI 1 trial than in the ULTRA2 trial and our trial may have reflected differences in trial design; patients 


\begin{tabular}{|c|c|c|}
\hline Event & $\begin{array}{l}\text { Adalimumab } \\
(\mathrm{N}=386)\end{array}$ & $\begin{array}{c}\text { Vedolizumab } \\
\qquad(\mathrm{N}=383)\end{array}$ \\
\hline \multicolumn{3}{|l|}{ Adverse events that occurred during the trial period $\dagger$} \\
\hline Any adverse event — no. of patients (\%) & $267(69.2)$ & $240(62.7)$ \\
\hline Mild & $118(30.6)$ & $111(29.0)$ \\
\hline Moderate & $109(28.2)$ & $92(24.0)$ \\
\hline Severe & $40(10.4)$ & 37 (9.7) \\
\hline Adverse events that led to discontinuation of a trial drug & $25(6.5)$ & $17(4.4)$ \\
\hline Adverse events, excluding ulcerative colitis — no. of patients (\%) & $250(64.8)$ & $229(59.8)$ \\
\hline Serious adverse events - no. of patients $(\%) \ddagger$ & $53(13.7)$ & $42(11.0)$ \\
\hline $\begin{array}{l}\text { Serious adverse events that led to discontinuation of a trial drug } \\
\qquad \text { - no. of patients (\%) }\end{array}$ & $13(3.4)$ & $10(2.6)$ \\
\hline Serious adverse events, excluding ulcerative colitis - no. of patients (\%) & $27(7.0)$ & $28(7.3)$ \\
\hline Death — no. of patients (\%) & 0 & $1(0.3) \int$ \\
\hline \multicolumn{3}{|l|}{ Exposure-adjusted incidence rates of adverse events 9} \\
\hline $\begin{array}{l}\text { Infections and infestations - no. of patients/incidence rate per } \\
\qquad 100 \text { patient-yr }\end{array}$ & $124 / 34.6$ & $103 / 23.4$ \\
\hline Clostridia & $2 / 0.6$ & $5 / 1.1$ \\
\hline Herpesvirus & $15 / 4.2$ & $2 / 0.5$ \\
\hline Lower respiratory tract & $7 / 2.0$ & $5 / 1.1$ \\
\hline Upper respiratory tract & $65 / 18.1$ & $55 / 12.5$ \\
\hline Serious infections and infestations & $8 / 2.2$ & $7 / 1.6$ \\
\hline $\begin{array}{l}\text { Musculoskeletal and connective-tissue disorders - no. of patients/ } \\
\text { incidence rate per } 100 \text { patient-yr }\end{array}$ & $44 / 12.3$ & $50 / 11.4$ \\
\hline Arthralgia & $16 / 4.5$ & $18 / 4.1$ \\
\hline $\begin{array}{l}\text { Skin and subcutaneous-tissue disorders - no. of patients/incidence rate } \\
\text { per } 100 \text { patient-yr }\end{array}$ & $52 / 14.5$ & $38 / 8.6$ \\
\hline Psoriasis & $6 / 1.7$ & $1 / 0.2$ \\
\hline
\end{tabular}

* Adverse events were classified according to the Medical Dictionary for Regulatory Activities System Organ Class categorization and preferred terms, version 21.0, and were analyzed according to the treatment actually received in the safety population, which included all the patients who underwent randomization and received at least one dose of a trial drug. $\uparrow$ The trial period was the time from the first dose of a trial drug and up to 126 days after the last dose.

No cases of progressive multifocal leukoencephalopathy have been reported.

$\int$ The one death in the vedolizumab group was not considered by the site investigator to be related to the trial drug.

q The exposure-adjusted incidence rate (per 100 patient-years) was defined as the number of patients who had the adverse event divided by the total exposure time among the patients. The results included the final 68-week safety follow-up.

who had a clinical response underwent an additional randomization at week 6 in the GEMINI 1 trial, but the patients in the ULTRA2 trial and our trial underwent randomization only at baseline. In addition, the ULTRA2 trial and the GEMINI 1 trial included a higher percentage of patients who had previously received treatment with a TNF inhibitor than the VARSITY trial. Direct comparisons of efficacy between the clinical trials are difficult and further highlight the need for direct head-to-head trials such as the VARSITY trial.

The results of the current trial suggest that corticosteroid-free clinical remission occurred in a higher percentage of patients in the adalimumab group than in the vedolizumab group. It is difficult to explain the inconsistency of the results between this secondary remission outcome and the primary remission outcome. The trial did not require a specific schedule for corticoste- 
roid tapering, which can vary among practitioners. However, this limitation should not have resulted in differential effects in the two treatment groups.

There were no notable treatment differences between patients who were receiving concomitant immunomodulator therapy and those who were not. A previous pooled-analysis study suggested that the immunomodulator-adalimumab combination therapy did not provide efficacy benefits beyond adalimumab monotherapy. ${ }^{17}$

In the VARSITY trial, a subgroup of patients who had previous use of infliximab or golimumab were enrolled, and therefore the results observed among these patients in the adalimumab group reflect the efficacy in clinical practice among patients who switched to adalimumab from a drug within the same drug class. We might have postulated that adalimumab would be disadvantaged relative to vedolizumab for patients who previously received treatment with a TNF inhibitor; however, our findings did not suggest this.

Histologic remission was an exploratory outcome of this trial and was assessed with the Geboes score and the Robarts Histopathologic Index score. The results for the outcomes of histologic remission were consistent with the findings for clinical remission and endoscopic improvement.

Few differences were observed between the trial groups in terms of the most commonly reported adverse events. The exposure-adjusted incidence rate of infection was 23.4 per 100 patientyears in the vedolizumab group and 34.6 per 100 patient-years in the adalimumab group.

The double-blind, double-dummy nature of the trial meant that dose intensification in either treatment group was not practical if blinding was to be maintained. The dosing regimens selected for this trial were based on a conservative approach and use according to U.S. labels. Realworld studies have shown improved efficacy outcomes after dose intensification in both adalimumab and vedolizumab therapies..$^{18,19}$ Data from ongoing trials of adalimumab (ClinicalTrials .gov number, NCT02065622) and vedolizumab (NCT03029143) may further characterize the effect of higher doses on efficacy outcomes.

In conclusion, the results of our trial involving patients with moderately to severely active ulcerative colitis show the superiority of vedolizumab over adalimumab in terms of clinical remission and endoscopic improvement but not of corticosteroid-free clinical remission.

A data sharing statement provided by the authors is available with the full text of this article at NEJM.org.

Supported by Takeda.

Dr. Sands reports receiving consulting fees from 4D Pharma, AbbVie, Amgen, AstraZeneca, Capella Bioscience, Celltrion Healthcare, EnGene, Ferring, Janssen Global Services, Lyndra, MedImmune, Oppilan Pharma, Otsuka America Pharmaceutical, Palatin Technologies, Progenity, Prometheus Laboratories, Protagonist Therapeutics, Rheos Medicines, Seres Therapeutics, Sienna Biopharmaceuticals, Synergy Pharmaceuticals, TiGenix, UCB, Valeant Pharmaceuticals North America, and Vivelix Pharmaceuticals, consulting fees and advisory board fees from Allergan, Arena Pharmaceuticals, Boehringer Ingelheim, Eli Lilly and Company, F. Hoffmann-La Roche, and Shire, grant support and advisory board fees from Celgene Corporation, advisory board fees from Ironwood Pharmaceuticals and Target PharmaSolutions, grant support, consulting fees, and advisory board fees from Janssen Biotech, grant support, consulting fees, advisory board fees, and lecture fees from Pfizer, grant support and consulting fees from Theravance Biopharma, and consulting fees and lecture fees from Gilead Sciences; Dr. Peyrin-Biroulet, receiving grant support, consulting fees, lecture fees, and advisory board fees from AbbVie, Takeda, and MSD, consulting fees, lecture fees, and advisory board fees from Janssen, Ferring, Tillots, Celltrion, Pfizer, and Roche, consulting fees and advisory board fees from Genentech, Pharmacosmos, Sandoz, Celgene, Allergan, Arena, Gilead, and Amgen, consulting fees from Boehringer Ingelheim, Index Pharmaceuticals, and Alma, consulting fees and lecture fees from Biogen and Samsung Bioepis, advisory board fees from Sterna, Nestle, and Enterome, lecture fees from Hikma, and holding stock options in CT-SCOUT; Dr. Loftus, receiving grant support and consulting fees from Takeda, Janssen, AbbVie, UCB, Pfizer, Amgen, Genentech, Gilead, Seres Therapeutics, and Celgene/Receptos, grant support, consulting fees, and fees for serving on a steering committee from Gilead, consulting fees and fees for serving on a data and safety monitoring board from Eli Lilly, consulting fees from Celltrion Healthcare, Bristol-Myers Squibb, Boehringer Ingelheim, Allergan, CVS Caremark, and Napo Pharmaceuticals, grant support from MedImmune and Robarts Clinical Trials, and fees for serving on a data and safety monitoring board from Mesoblast; Dr. Danese, receiving consulting fees from AbbVie, Allergan, Amgen, AstraZeneca, Biogen, Boehringer Ingelheim, Celgene, Celltrion, Ferring, Gilead, Hospira, Janssen, Johnson \& Johnson, MSD, Mundipharma, Pfizer, Roche, Sandoz, Takeda, TiGenix, UCB, and Vifor, and advisory board fees from Arena; Dr. Colombel, receiving grant support, consulting fees, advisory board fees, and lecture fees from AbbVie, Janssen, and Takeda, consulting fees, advisory board fees, and lecture fees from Amgen, Ferring, Shire, and Allergan, consulting fees and advisory board fees from Arena, Boehringer Ingelheim, Celgene, Celltrion, Eli Lilly, Enterome, Genentech, Ipsen, Landos, MedImmune, Merck, Novartis, Pfizer, TiGenix and Viela, and holding stock options in Intestinal Biotech Development and Genfit; Dr. Törüner, advisory board fees and lecture fees from AbbVie, MSD, and UCB, lecture fees from Ferring and Sandoz, consulting fees and advisory board fees from Celltrion and Pfizer, and consulting fees, lecture fees, and advisory board fees from Takeda and Janssen; Dr. Jonaitis, receiving fees for serving as research coordinator and lecture fees from AbbVie, lecture fees from Ferring, KRKA, and Genentech, and travel support and conference registration reimbursement from Takeda; Drs. Abhyankar, Chen, Rogers, 
Lirio, and Bornstein, being employed by Takeda; and Dr. Schreiber, receiving advisory board fees from AbbVie, Arena, BMS, Biogen, Celltrion, Celgene, IMAB, Gilead, MSD, Mylan, Pfizer, Fresenius, Janssen, Takeda, Theravance, Provention Bio, Protagonist, and Falk. No other potential conflict of interest relevant to this article was reported.

Disclosure forms provided by the authors are available with the full text of this article at NEJM.org.
We thank the patients who participated in the trial, their caregivers, and the trial investigators, members of the VARSITY data and safety monitoring board and adjudication committee, and members of the VARSITY trial team. We also thank Margo Jaffee, Amanda Tweed, Sharon Hunter, Nick Brown, and Theresa Peterson for contributing their expertise and support and Vinay Pasupuleti of ProEd Communications, for providing medical writing support (funded by Takeda).

\section{REFERENCES}

1. Ungaro $\mathrm{R}$, Mehandru $\mathrm{S}$, Allen $\mathrm{PB}$ Peyrin-Biroulet L, Colombel JF. Ulcerative colitis. Lancet 2017;389:1756-70.

2. Harbord M, Eliakim R, Bettenworth $\mathrm{D}$, et al. Third European evidence-based consensus on diagnosis and management of ulcerative colitis. Part 2: current management. J Crohns Colitis 2017;11:769-84. 3. Rubin DT, Ananthakrishnan AN, Siegel CA, Sauer BG, Long MD. ACG clinical guideline: ulcerative colitis in adults. Am J Gastroenterol 2019;114:384-413.

4. Gordon KB, Callis Duffin K, Bissonnette $\mathrm{R}$, et al. A phase 2 trial of guselkumab versus adalimumab for plaque psoriasis. N Engl J Med 2015;373:136-44.

5. Taylor PC, Keystone EC, van der Heijde $\mathrm{D}$, et al. Baricitinib versus placebo or adalimumab in rheumatoid arthritis. N Engl J Med 2017;376:652-62.

6. Wyant T, Fedyk E, Abhyankar B. An overview of the mechanism of action of the monoclonal antibody vedolizumab. J Crohns Colitis 2016;10:1437-44.

7. Lamb CA, O'Byrne S, Keir ME, Butcher EC. Gut-selective integrin-targeted therapies for inflammatory bowel disease. J Crohns Colitis 2018;12:Suppl 2:S653-S668.

8. D'Haens G, Sandborn WJ, Feagan BG, et al. A review of activity indices and efficacy end points for clinical trials of medical therapy in adults with ulcerative colitis. Gastroenterology 2007;132:763-86. 9. Irvine EJ. Development and subsequent refinement of the Inflammatory Bowel Disease Questionnaire: a qualityof-life instrument for adult patients with inflammatory bowel disease. J Pediatr Gastroenterol Nutr 1999;28:S23-S27.

10. Lewis JD, Chuai S, Nessel L, Lichtenstein GR, Aberra FN, Ellenberg JH. Use of the noninvasive components of the Mayo score to assess clinical response in ulcerative colitis. Inflamm Bowel Dis 2008;14: 1660-6.

11. Jauregui-Amezaga A, Geerits A, Das Y, et al. A simplified Geboes Score for ulcerative colitis. J Crohns Colitis 2017;11:305-13. 12. Mosli MH, Feagan BG, Zou G, et al. Development and validation of a histological index for UC. Gut 2017;66:50-8.

13. Cui L, Hung HM, Wang SJ. Modification of sample size in group sequential clinical trials. Biometrics 1999;55:853-7. 14. Mehta CR, Pocock SJ. Adaptive increase in sample size when interim results are promising: a practical guide with examples. Stat Med 2011;30:3267-84.
15. Sandborn WJ, van Assche G, Reinisch $\mathrm{W}$, et al. Adalimumab induces and maintains clinical remission in patients with moderate-to-severe ulcerative colitis. Gastroenterology 2012;142(2):257-65.e1.

16. Feagan BG, Rutgeerts P, Sands BE, et al. Vedolizumab as induction and maintenance therapy for ulcerative colitis. N Engl J Med 2013;369:699-710.

17. Colombel JF, Jharap B, Sandborn WJ, et al. Effects of concomitant immunomodulators on the pharmacokinetics, efficacy and safety of adalimumab in patients with Crohn's disease or ulcerative colitis who had failed conventional therapy. Aliment Pharmacol Ther 2017;45:50-62.

18. Van de Vondel S, Baert F, Reenaers C, et al. Incidence and predictors of success of adalimumab dose escalation and deescalation in ulcerative colitis: a realworld Belgian cohort study. Inflamm Bowel Dis 2018;24:1099-105.

19. Schreiber S, Dignass A, Peyrin-Biroulet $\mathrm{L}$, et al. Systematic review with metaanalysis: real-world effectiveness and safety of vedolizumab in patients with inflammatory bowel disease. J Gastroenterol 2018;53:1048-64

Copyright (C) 2019 Massachusetts Medical Society.

Visit the article page at NEJM.org and click on Metrics for a dashboard that logs views, citations, media references, and commentary. NEJM.org/about-nejm/article-metrics. 